\title{
Activity Status
}

National Cancer Institute

\section{Source}

National Cancer Institute. Activity Status. NCI Thesaurus. Code C70711.

A state of an activity in relation to its execution, timing, objective(s) or other quantitative or qualitative parameter(s). 